\title{
Integral Eigen-Pair Balanced Classes of Graphs with Their Ratio, Asymptote, Area, and Involution-Complementary Aspects
}

\author{
Paul August Winter and Carol Lynne Jessop \\ Department of Mathematics, Howard College, University of KwaZulu-Natal, Glenwood, Durban 4041, South Africa \\ Correspondence should be addressed to Paul August Winter; winterp@ukzn.ac.za
}

Received 26 May 2014; Accepted 3 September 2014; Published 23 September 2014

Academic Editor: Cai Heng Li

Copyright ( 2014 P. A. Winter and C. L. Jessop. This is an open access article distributed under the Creative Commons Attribution License, which permits unrestricted use, distribution, and reproduction in any medium, provided the original work is properly cited.

\begin{abstract}
The association of integers, conjugate pairs, and robustness with the eigenvalues of graphs provides the motivation for the following definitions. A class of graphs, with the property that, for each graph (member) of the class, there exists a pair $a, b$ of nonzero, distinct eigenvalues, whose sum and product are integral, is said to be eigen-bibalanced. If the ratio $(a+b) /(a \cdot b)$ is a function $f(n)$, of the order $n$ of the graphs in this class, then we investigate its asymptotic properties. Attaching the average degree to the Riemann integral of this ratio allowed for the evaluation of eigen-balanced areas of classes of graphs. Complete graphs on $n$ vertices are eigen-bibalanced with the eigen-balanced ratio $(n-2) /(1-n)=f(n)$ which is asymptotic to the constant value of -1 . Its eigenbalanced area is $(n-1)(n-\ln (n-1))$-we show that this is the maximum area for most known classes of eigen-bibalanced graphs. We also investigate the class of eigen-bibalanced graphs, whose class of complements gives rise to an eigen-balanced asymptote that is an involution and the effect of the asymptotic ratio on the energy of the graph theoretical representation of molecules.
\end{abstract}

\section{Introduction: Integers, Conjugate Pairs, and Eigenvalues of a Graph}

The graphs in this paper are simple and connected and all graph properties mentioned will be according to definitions in Harris et al. [1]. There has been much work done in the analysis of eigenvalues of matrices which are adjacency matrices of associated graphs. The following are some examples of these findings, with the references as specified.

(i) There has been interest in classes of graphs whose pairs of eigenvalues satisfy certain conditions. In Sarkar and Mukherjee [2], graphs are considered with reciprocal pairs of eigenvalues $(\lambda, 1 / \lambda)$ whose product is the integer 1.

(ii) Pairs of eigenvalues $(1,-1)$, summing to 0 , and whose product is -1 , are considered in Dias [3].

(iii) Summing the eigenvalues of the adjacency matrix of a graph is connected to the energy of physical structures; see Aimei et al. [4].

(iv) In the paper by van Dam [5], on regular graphs with 4 eigenvalues, he considers the eigenvalue pair of real conjugates $(a \pm \sqrt{b}) / 2$ and shows that if a matrix has an eigenvalue $(a+\sqrt{b}) / 2$, then it has an eigenvalue $(a-$ $\sqrt{b}) / 2$ of the same multiplicity, and vice versa. Adding the pair of conjugates $(a+\sqrt{b}) / 2$ and $(a-\sqrt{b}) / 2$, we obtain the integer $a$. Their product is $\left(a^{2}-b\right) / 4$ which is integral, provided the numerator is a multiple of 4 . The paper shows that there are graphs whose matrices have conjugate pairs of eigenvalues whose sum does not necessarily sum to the same integer $a$.

The following references show other areas of research which, together with the results on eigenvalues, provide motivation for the new definitions which are contained in this paper.

(i) There has been interest in the importance of pairs of numbers, whose sum and product produce the same integral constant, and this exists outside the linear algebra of matrices; see, for example, Dias [3].

(ii) In Brouwer and Haemers [6], integral trees (where the eigenvalues of trees are integral) are investigated. 
(iii) In the cryptography article, Hamada [7] considers the conjugate code pair consisting of linear codes $\left[n, k^{\prime}\right]$ and $\left[n, k^{\prime \prime}\right]$ satisfying the constant (integral) sum term $k^{\prime}+k^{\prime \prime}=n+k$, where $n$ is the dimension of the vector space involved and $k$ is the $k$-digit secret information sent.

(iv) In the paper by Kadin [8] he investigates the Cooper pair of opposite wave vectors $k$ and $-k$ which balance by summing to 0 and whose product is $-k^{2}$.

(v) Hinch and Leal [9] consider the notion of an isolated particle in the absence of rotary Brownian motion, under the condition that the hydrodynamic and external field couples exactly balance one another.

(vi) Armstrong [10] investigates the importance of the quadratic part of a characteristic equation which has the form $x^{2}-\tau x+\delta$. This quadratic gives rise to the two eigenvalues $a, b=\left(\tau \pm \sqrt{\tau^{2}-4 \delta}\right) / 2$. The sum and product $(\tau, \delta$, resp.) are often referred to as the eigenpair, but we will focus on the pair of eigenvalues $(a, b)$ as the eigen-pair.

Generally, there often exist two eigenvalues (associated with the adjacency matrix of a graph) whose sum or product is integral It is therefore possible to get the same integer when adding or multiplying two distinct, nonzero eigenvalues. This integer is either a fixed constant or a function of an inherent property of the graph, for all graphs belonging to a certain class of graphs. For example, complete graphs $K_{n}$ on $n$ vertices have a pair of eigenvalues with sum of $f(n)=n-2$ and product of $g(n)=1-n$ for each $n \geq 2$, and the complete bipartite graphs $K_{n / 2, n / 2}$ on $n$ vertices have eigen-pair sum (of nonzero eigenvalues) of 0 and product of $-n^{2} / 4$.

Definition 1 (function $f(p)$ of a member of a class of graphs). We define a function of a member belonging to a class of graphs as a real function $f(p)$ of an inherent property $p$ of the member in the class, such as the number of vertices or the clique number of a graph, and so forth.

In this paper, we combine the ideas of a pair of eigenvalues and their balanced integral sum and product with respect to a class of graphs, to introduce a definition which is a form of integral-eigenvalue balance associated with classes of graphs. We investigate classes of graphs on $n$ vertices with pairs $(a, b)$ of distinct nonzero eigenvalues such that $a+b=s$ or $a b=t$, where $s, t$ are the same integer (resp.) for each graph in the class or the same function for each graph in the class.

\section{Integral Sum Eigen-Pair Balanced Classes of Graphs}

Definition $2\left(\operatorname{sum}^{*}(s)^{*}\right.$ eigen-pair (integral) balanced). The class $\mathfrak{I}$ of connected graphs on $n$ elements is said to be sum $^{*}(s)^{*}$ eigen-pair (integral) balanced if there exists a pair $(a, b)$ of distinct nonzero eigenvalues of the matrices associated with each class of the structures such that $a+b=s$ is the same integer as a fixed constant for each member in the class or $s$ is the same integer as a function of each member in the class. The sum balance is exact if $s$ is the same integer as a fixed constant for each member in the class, or otherwise it is nonexact.

The following are some examples of such classes of graphs, noting that $\operatorname{sum}(a, b)=a+b$ in the examples below.

2.1. Complete Graphs. The distinct eigenvalues of the complete graph $K_{n}$ are -1 and $(n-1)$ (as per Brouwer and Haemers [6]), with the sum of the eigenvalues being ( $n-$ 2 ). Therefore the class of complete graphs $K_{n}$ is nonexact sum $^{*}(n-2) *$ eigen-pair balanced, for $n \geq 3$.

2.2. Complete Bipartite Graphs. The class of complete bipartite graphs $K_{p, p}$ on $n=2 p$ vertices has as its associated eigenvalues $p,-p$, and 0 (as per Brouwer and Haemers [6]), so it is exact $\operatorname{sum}^{*}(0)^{*}$ eigen-pair balanced.

The class of complete bipartite graphs $K_{p, k}$ on $p+k$ vertices, $p \neq k$, has eigenvalues $-\sqrt{p k}, \sqrt{p k}, 0$ (as per Brouwer and Haemers [6]), so it is exact $\operatorname{sum}^{*}(0)^{*}$ eigen-pair balanced (this includes the star graphs with radius 1 ).

2.3. Cycle Graphs. From Brouwer and Haemers [6], the cycle $C_{n}$ on $n$ vertices and $n$ edges has eigenvalues

$$
2 \cos \left(\frac{2 \pi j}{n}\right), \quad 0 \leq j \leq n-1 .
$$

The 3-cycle (complete graph on 3 vertices) has distinct eigenvalues -1 and 2 .

The 4-cycle has distinct eigenvalues 2,0 , and -2 .

The 5 -cycle has distinct eigenvalues $2,(-1+\sqrt{5}) / 2$, and $(-1-\sqrt{5}) / 2$.

The 6-cycle has distinct eigenvalues $2,1,-1$, and -2 .

The 7-cycle has distinct eigenvalues $2,1.247,-0.445$, and 1.802 .

For the 3-, 5-, and 6-cycle, there exist two distinct nonzero eigenvalues whose sum is 1 . However, for the 7-cycle, there are no two distinct eigenvalues whose sum is 1 . Therefore the class of cycles is not sum(1)eigen-pair balanced as it does not satisfy Definition 2.

However, even cycles are $\operatorname{sum}^{*}(0)^{*}$ eigen-pair balanced, since if $n=2 k$,

$$
2 \cos \left(\frac{2 \pi j}{n}\right)=2 \cos \left(\frac{\pi j}{k}\right), \quad 0 \leq j \leq 2 k-1 .
$$

Then $j=0$ and $j=k$ yield eigenvalues of 2 and -2 , respectively.

2.4. Path Graphs. From Brouwer and Haemers [6], the path $P_{n}$ on $n \geq 2$ vertices and $(n-1)$ edges has eigenvalues

$$
2 \cos \left(\frac{\pi j}{n+1}\right), \quad 1 \leq j \leq n .
$$

Note that

$$
\begin{aligned}
\cos \left(\frac{n \pi}{n+1}\right)= & \cos \left(\pi-\frac{\pi}{n+1}\right)=\cos \pi \cos \left(\frac{\pi}{n+1}\right) \\
& +\sin \pi \sin \left(\frac{\pi}{n+1}\right)=-\cos \left(\frac{\pi}{n+1}\right)
\end{aligned}
$$


so that the nonzero pair, with $j=n$ and $j=1$,

$$
(a, b)=\left(2 \cos \left(\frac{n \pi}{n+1}\right), 2 \cos \left(\frac{\pi}{n+1}\right)\right)
$$

has the sum

$$
\begin{aligned}
\operatorname{sum}(a, b) & =2 \cos \left(\frac{n \pi}{n+1}\right)+2 \cos \left(\frac{\pi}{n+1}\right) \\
& =-2 \cos \left(\frac{\pi}{n+1}\right)+2 \cos \left(\frac{\pi}{n+1}\right)=0,
\end{aligned}
$$

so the class of path graphs is exact $\operatorname{sum}^{*}(0)^{*}$ eigen-pair balanced.

2.5. Graph Which Is the Join of Two Graphs Whose Adjacency Matrices Are Both Circulant Matrices. From Lee and Yeh [11], the conjugate eigenvalues of the join of two circulant matrices of graphs are

$$
(a, b)=n\left[\frac{-\left(d_{A}-d_{B}\right) \pm \sqrt{\left(d_{A}-d_{B}\right)^{2}+4 n m}}{2 n}\right]+d_{A} .
$$

So the sum of the eigenvalues is

$$
\begin{aligned}
\operatorname{Sum}(a, b)= & n\left[\frac{-\left(d_{A}-d_{B}\right)+\sqrt{\left(d_{A}-d_{B}\right)^{2}+4 n m}}{2 n}\right]+d_{A} \\
& +n\left[\frac{-\left(d_{A}-d_{B}\right)-\sqrt{\left(d_{A}-d_{B}\right)^{2}+4 n m}}{2 n}\right]+d_{A} \\
= & n\left[\frac{-2\left(d_{A}-d_{B}\right)}{2 n}\right]+2 d_{A} \\
= & -\left(d_{A}-d_{B}\right)+2 d_{A} \\
= & d_{A}+d_{B},
\end{aligned}
$$

so the class of graphs, which are the join of two graphs whose adjacency matrices are circulant, is $\operatorname{sum}^{*}\left(d_{A}+d_{B}\right)^{*}$ eigen-pair balanced.

2.6. Wheel Graphs. As per Lee and Yeh [11], the wheel graph $W_{n}$ on $n$ vertices, with $(n-1)$ spokes (join of cycle with a single vertex), has conjugate eigenvalues

$$
(a, b)=\frac{2 \pm \sqrt{4+4(n-1)}}{2} .
$$

The sum of the conjugate eigenvalues is therefore

$$
\begin{aligned}
\operatorname{sum}(a, b) & =\frac{2+\sqrt{4+4(n-1)}}{2}+\frac{2-\sqrt{4+4(n-1)}}{2} \\
& =2
\end{aligned}
$$

so the class of cycle wheel graphs is exact sum ${ }^{*}(2)^{*}$ eigen-pair balanced.
2.7. Strongly Regular Graphs. If a connected regular graph $G$ of degree $k$ with parameters $(k, \lambda, \mu)$ is strongly regular (i.e., $\operatorname{srg}(\nu, k, \lambda, \mu))$, then $A(G)$ has at least 3 different eigenvalues. The eigenvalues are

$$
\begin{gathered}
k, \frac{(\lambda-\mu)+\sqrt{(\lambda-\mu)^{2}-4(k-\mu)}}{2}, \\
\frac{(\lambda-\mu)-\sqrt{(\lambda-\mu)^{2}-4(k-\mu)}}{2} .
\end{gathered}
$$

See Spielman [12].

The complement of an $\operatorname{srg}(\nu, k, \lambda, \mu)$ is also strongly regular. It is an $\operatorname{srg}(v, v-k-1, v-2-2 k+\mu, v-2 k+\lambda)$.

Note that if we ignore the largest eigenvalue $k$ of strongly regular graphs, adding the remaining two eigenvalues yields the integer $(\lambda-\mu)$ so the class of strongly regular graphs with parameters $(\nu, k, \lambda, \mu)$ is nonexact $\operatorname{sum}^{*}(\lambda-\mu)^{*}$ eigenpair balanced; see Godsil and Royle [13] for more information on strongly regular graphs.

\subsection{Divisible Design Graphs}

Definition 3 (divisible design graph). A $k$-regular graph is a divisible design graph if the vertex set can be partitioned into $m$ classes of size $n$, such that two distinct vertices from the same class have exactly $\lambda_{1}$ common neighbours, and two vertices from different classes have exactly $\lambda_{2}$ common neighbours.

The eigenvalues of divisible design graphs are provided in Haemers [14]; there are 5 distinct eigenvalues. Two of the eigenvalues are

$$
(a, b)= \pm \sqrt{k-\lambda_{1}}
$$

so the sum of the eigen-pair is

$$
\operatorname{sum}(a, b)=\left(\sqrt{k-\lambda_{1}}\right)+\left(-\sqrt{k-\lambda_{1}}\right)=0
$$

Therefore, the class of divisible design graphs is exact $\operatorname{sum}^{*}(0)^{*}$ eigen-pair balanced.

2.9. Hypercube Graphs. As per Brouwer and Haemears [6], the $p$-regular hypercube on $2^{p}$ vertices and $p 2^{p-1}$ edges has eigenvalues

$$
(p-2 k)^{\left(\begin{array}{l}
p \\
k
\end{array}\right)} ; \quad k=0,1,2, \ldots, p .
$$

Using the eigenvalues $p$ and $p-2 k$, for $p \neq 2 k$ and $k \neq 0$, this class of graphs will be sum* ${ }^{*}(2 p-2 k)^{*}$ eigen-pair balanced.

2.10. Eigenvalue Pair of Real Conjugates. The following results are due to van Dam and Haemers [15].

By $Z[x]$ and $Q[x]$ we denote the rings of polynomials over the integer and rational numbers, respectively.

Lemma 4. If a monic polynomial $p(x) \in Z[x]$ has a monic divisor $q(x) \in Q[x]$, then also $q(x) \in Z[x]$. 
Lemma 5. If $(a+\sqrt{b})$, with $a, b \in Q$, is an irrational root of a polynomial $p(x) \in Q[x]$, then so is $(a-\sqrt{b})$, with the same multiplicity.

The characteristic polynomial $P_{A(G)}(\lambda)$ of the adjacency matrix of a graph is monic and has integral coefficients. Using Lemmas 4 and 5 we now obtain the following results.

Corollary 6. Every rational eigenvalue of a graph is integral.

Corollary 7. If $(a+\sqrt{b}) / 2$ is an irrational eigenvalue of $a$ graph, for some $a, b \in Q$, then so is $(a-\sqrt{b}) / 2$, with the same multiplicity, and $a, b \in Z$.

Adding the pair of real conjugates $(a+\sqrt{b}) / 2,(a-\sqrt{b}) / 2$ we obtain the integer $a$.

Therefore, if the real conjugate pairs are eigenvalues associated with the adjacency matrix of all graphs belonging to a class of graphs, then the class of graphs is $\operatorname{sum}^{*}(a)^{*}$ eigenpair balanced.

\section{Integral Product Eigen-Pair Balanced Classes of Graphs}

Definition 8 (product* $(t)^{*}$ eigen-pair (integral) balanced). A class $\mathfrak{I}$ of connected graphs on $n$ elements is said to be product $^{*}(t)^{*}$ eigen-pair (integral) balanced if there exists a pair of $(a, b)$ of distinct nonzero eigenvalues (counting eigenvalues only once, i.e., ignoring multiplicities) of the matrices associated with each class of the structures such that $(a b=t)$ is the same integer as a fixed constant for each member in the class or $t$ is the same integer as a function of each member in the class. The product balance is exact if $t$ is the same integer as a fixed constant for each member in the class; otherwise it is nonexact.

The following are some examples of such classes of product balanced classes of graphs, discussed above, noting that the product of the pair $(a, b)=a b$ in the examples below.

3.1. Complete Graphs. The class of complete graphs $K_{n}$ is nonexact product ${ }^{*}(1-n)^{*}$ eigen-pair balanced for $n \geq 3$, since the eigenvalues of the associated adjacency matrices, are, as per Section 2.1, -1 and $(n-1)$.

3.2. Complete Bipartite Graphs. The class of complete bipartite graphs $K_{p, p}$ on $n=2 p$ vertices has as its associated eigenvalues $p,-p$, and 0 (as per Section 2.2), so that they are nonexact product ${ }^{*}\left(-p^{2}\right)^{*}$ eigen-pair balanced.

The class of complete bipartite graphs $K_{p, k}$ on $(p+k)$ vertices, $p \neq k$, has distinct eigenvalues $-\sqrt{p k}, \sqrt{p k}$, and 0 (as per Section 2.2), so that it is nonexact product* $(-p k)^{*}$ eigenpair balanced (this includes the star graphs with radius 1 ).

3.3. Cycle Graphs. Cycles graphs on $n$ vertices have associated eigenvalues

$$
2 \cos \left(\frac{2 \pi j}{n}\right), \quad 0 \leq j \leq n-1
$$

$\mathrm{C}_{3}$ on 3 vertices has eigenvalues 2 and -1 so that the eigenpair product is -2 .

$\mathrm{C}_{4}$ on 4 vertices has eigenvalues 2,0 , and -2 so that the eigen-pair product is -4 .

$C_{5}$ on 5 vertices has eigenvalues $2,(-1+\sqrt{5}) / 2$, and $(-1-$ $\sqrt{5}) / 2$ with conjugate eigen-pair product -1 .

$C_{6}$ on 6 vertices has eigenvalues $2,1,-1$, and -2 . Possible eigen-pair products are $-1,-2,2$, and -4 .

The 7 -cycle has eigenvalues $2,1.247,-0.445$, and -1.802 . No product of two eigenvalues yields an integer!

Therefore, the class of cycles is not eigen ${ }^{*}(k)^{*}$ product balanced for any integer $k$.

However, the class of even cycles is product ${ }^{*}(-4)^{*}$ eigenpair balanced, since if $n=2 k$ then

$$
2 \cos \left(\frac{2 \pi j}{n}\right)=2 \cos \left(\frac{2 \pi j}{2 k}\right) ; \quad 0 \leq j \leq 2 k-1,
$$

so that for $j=0$ we get eigenvalue 2 and for $j=k$ we get eigenvalue -2 , with eigen-pair product -4 .

3.4. Path Graphs. Paths graphs on $n$ vertices have eigenvalues:

$$
2 \cos \left(\frac{\pi j}{n+1}\right), \quad 1 \leq j \leq n .
$$

From Section 2.4,

$$
2 \cos \left(\frac{\pi n}{n+1}\right)=-2 \cos \left(\frac{\pi}{n+1}\right)
$$

so that with $j=1$ and $(j=n)$ the product is

$$
-2\left(1+\cos \left(\frac{2 \pi}{n+1}\right)\right)
$$

which is a function of $n$ but is not integral in general.

3.5. Graph Which Is the Join of Two Graphs Whose Adjacency Matrices Are Both Circulant Matrices. The conjugate eigenvalues are

$$
(a, b)=n\left[\frac{-\left(d_{A}-d_{B}\right) \pm \sqrt{\left(d_{A}-d_{B}\right)^{2}+4 n m}}{2 n}\right]+d_{A} .
$$

So the product of the eigenvalues is

$$
\begin{aligned}
& \text { product }(a, b) \\
& =\left\{n\left[\frac{-\left(d_{A}-d_{B}\right)+\sqrt{\left(d_{A}-d_{B}\right)^{2}+4 n m}}{2 n}\right]+d_{A}\right\} \\
& \times\left\{n\left[\frac{-\left(d_{A}-d_{B}\right)-\sqrt{\left(d_{A}-d_{B}\right)^{2}+4 n m}}{2 n}\right]+d_{A}\right\}
\end{aligned}
$$




$$
\begin{aligned}
= & n^{2}\left[\frac{\left(d_{A}-d_{B}\right)^{2}-\left[\left(d_{A}-d_{B}\right)^{2}+4 n m\right]}{4 n^{2}}\right] \\
& -2 d_{A}\left(d_{A}-d_{B}\right)+d_{A}^{2} \\
= & {\left[\frac{-(4 n m)}{4}\right]-2 d_{A}\left(d_{A}-d_{B}\right)+d_{A}^{2} } \\
= & -m n-2 d_{A}\left(d_{A}-d_{B}\right)+d_{A}^{2},
\end{aligned}
$$

so the class of graphs whose adjacency matrix is the join of two graphs whose adjacency matrices are circulant matrices is product ${ }^{*}\left(-m n-2 d_{A}\left(d_{A}-d_{B}\right)+d_{A}^{2}\right)^{*}$ eigen-pair balanced.

3.6. Wheel Graphs. The wheel graph $W_{n}$ on $n$ vertices, with $(n-1)$ spokes, has conjugate eigenvalues

$$
(a, b)=\frac{2 \pm \sqrt{4+4(n-1)}}{2} .
$$

The product of the conjugate eigenvalues is

$$
\begin{aligned}
\operatorname{product}(a, b) & =\left[\frac{2+\sqrt{4+4(n-1)}}{2}\right]\left[\frac{2-\sqrt{4+4(n-1)}}{2}\right] \\
& =\frac{4-[4+4(n-1)]}{4} \\
& =-(n-1),
\end{aligned}
$$

so the class of cycle wheel graphs is nonexact product ${ }^{*}(1-$ $n)^{*}$ eigen-pair balanced.

3.7. Strongly Regular Graphs. The conjugate eigen-pair of strongly regular graphs is

$$
(a, b)=\frac{(\lambda-\mu) \pm \sqrt{(\lambda-\mu)^{2}-4(k-\mu)}}{2} .
$$

If we multiply the two conjugate pairs of strongly regular graphs we obtain the integer $(\mu-k)$, so that the class of strongly regular graphs is nonexact $\operatorname{product}^{*}(\mu-k)^{*}$ eigenpair balanced.

3.8. Divisible Design Graphs. Two of the eigenvalues of a divisible design graph are

$$
(a, b)= \pm \sqrt{k-\lambda_{1}} .
$$

This class of graphs has eigen-pair product

$$
\begin{aligned}
\operatorname{product}(a, b) & =\left(\sqrt{k-\lambda_{1}}\right)\left(-\sqrt{k-\lambda_{1}}\right) \\
& =-\left(k-\lambda_{1}\right)=\lambda_{1}-k .
\end{aligned}
$$

Therefore, the class of divisible design graphs is nonexact product $^{*}\left(\lambda_{1}-k\right)^{*}$ balanced.
3.9. Bipartite Graphs with Four Distinct Eigenvalues. The eigenvalues of a bipartite graph with four distinct eigenvalues are

$$
(a, b)= \pm \sqrt{k-\lambda}, \quad(c, d)= \pm k .
$$

Then product $(a, b)=-(k-\lambda)$ and product $(c, d)=-k^{2}$.

Therefore, incidence graphs of symmetric 2- $(v, k, l)$ designs are product $t^{*}$ eigen-pair balanced for $t=(\lambda-k)$ and $t=-k^{2}$ of the nonexact kind.

3.10. Hypercube Graphs. The class of $p$-regular hypercubes on $2^{p}$ vertices and $p 2^{p-1}$ edges has eigenvalues:

$$
(p-2 k)^{\left(\begin{array}{l}
p \\
k
\end{array}\right)}, \quad 0 \leq k \leq p .
$$

Using the eigenvalues $p$ and $p-2 k$, for $p \neq 2 k, k \neq 0$, this class of graphs is product ${ }^{*}\left(p^{2}-2 p k\right)^{*}$ eigen-pair balanced.

3.11. Eigenvalue Pair of Real Conjugates. The product of the real conjugate pair of eigenvalues $(a+\sqrt{b}) / 2$ and $(a-\sqrt{b}) / 2$ is $\left(a^{2}-b\right) / 4$. This is integral, provided the numerator is a multiple of 4 .

Therefore, if the real conjugate pairs are eigenvalues associated with the adjacency matrix of all graphs belonging to a class of graphs, then the class of graphs is product ${ }^{*}\left(\left(a^{2}-\right.\right.$ b) $/ 4)^{*}$ eigen-pair balanced.

\section{Eigen-Bibalanced Classes of Graphs}

Definition 9 (eigen-bibalanced classes of graphs). Classes of connected graphs, which are both sum and product eigenpair balanced, are said to be eigen-bibalanced with respect to the eigen-pair $(a, b)$. If this pair is unique to the class, then it is uniquely eigen-bibalanced. For example, the class of complete graphs is uniquely eigen-bibalanced with respect to the eigenpair $(n-1,-1)$.

The largest eigenvalue occurs in the eigen-pair associated with some classes of graphs discussed above. We observe the following.

(i) The only regular eigen-pair balanced graphs on 2 and 3 vertices are $K_{2}$ and $K_{3}$.

(ii) The 4-cycle is the same as the complete bipartite graph $K_{2,2}$, which is sum and product eigen-pair balanced.

(iii) The only other regular graph on 4 vertices is $K_{4}$.

(iv) The 5 -cycle has eigenvalues $(2)^{1},((-1+\sqrt{5}) / 2)^{2}$, and $((-1-\sqrt{5}) / 2)^{2}$ which are not sum or product eigenpair balanced when the largest eigenvalue is included in the eigen-pair.

(v) The only other regular graph on 5 vertices is $K_{5}$.

Thus we have the following theorem.

Theorem 10. The only regular graphs on $n$ vertices, where $2 \leq$ $n \leq 5$, belonging to eigen-pair balanced classes of graphs, where the eigen-pair contains the largest eigenvalue, are $K_{2}, K_{3}, K_{4}$, $K_{5}$, and $K_{2,2}$. 


\section{Eigen-Bibalanced Classes of Graphs: Criticality, Ratios, Asymptotes, and Area}

If a class of connected graphs $\mathfrak{\Im}$ are both sum and product eigen-pair balanced with respect to the eigen-pair $(a, b)$, they have been defined above as eigen-bibalanced with respect to $(a, b)$. The class of complete graphs $G$ is eigen-bibalanced with the property that the removal of any vertex $v$ from $G$ results in a complete graph, which belongs to the same class of complete graphs, which is eigen-bibalanced. The same holds for complete bipartite graphs except for star graphs. Such graphs are said to be stable eigen-bibalanced.

Definition 11 (critically eigen-bibalanced classes of graphs). If $G$ belongs to a class $\mathfrak{I}$ of eigen-bibalanced graphs, and there exists a vertex $v$ of $G$, such that $G-v$ belongs to a class $\mathfrak{I}^{\prime}$ of graphs which is not eigen-bibalanced, we say that $\mathfrak{I}$ is critically eigen-bibalanced with respect to $v$.

Wheels on $p$ spokes are eigen-bibalanced and the removal of the central vertex results in $p$-cycles, which are not eigenbibalanced. Therefore, the class of wheel graphs is critically eigen-bibalanced with respect to its central vertex. This suggests that the central vertex is essential to the eigenbibalanced characteristic of wheels.

The reciprocals of eigenvalues are connected to the idea of robustness or tightness of graphs, Brouwer and Haemers [7]. Since $a$ and $b$ are nonzero, the sum of their reciprocals is defined. Therefore we have the following definition.

Definition 12 (eigen-bibalanced ratio of classes of graphs). The importance of ratios in graphs is well researched (see Winter and Adewusi [16]). The eigen-bibalanced ratio of the class of graphs (with respect to the eigen-pairs $(a, b)$ ) is

$$
r(a \Im b)=\frac{1}{b}+\frac{1}{a}=\frac{a+b}{a b} .
$$

As $a$ and $b$ are nonzero, the product $a b$ is never zero, and so this ratio will always be defined.

Definition 13 (eigen-bibalanced ratio asymptote of classes of graphs). If this ratio is a function $f(n)$ of the order $n$ of the graph and has a horizontal asymptote (see Winter and Adewusi [16]), we call this asymptote the eigen-bibalanced ratio asymptote with respect to the eigen-pair $(a, b)$ and is denoted by

$$
r(a \Im b)^{\infty} \text { or asymp }(r(a \Im b)) .
$$

This asymptote can be seen as describing the behavior of the ratio as the order of the graph becomes increasingly large.

The "area" term $n^{2}$ can be found in the following relation involving spanning trees. Let $D$ be the matrix composed of the degrees of $G$ in the main diagonal form $D^{\prime}$ by adding 1 to each entry of $D$. We then form the shadow number of a graph defined by $\operatorname{shad}(G)=\operatorname{det}\left(D^{\prime}-A\right)$, where $A$ is the adjacency matrix of $G$. We then have the combinatorial result

$$
n^{2}=\frac{\operatorname{shad}(G)}{t(G)}=\frac{\operatorname{det}\left(D^{\prime}-A\right)}{t(G)},
$$

where $t(G)$ is the number of spanning trees of a connected graph $G$.

Also, the number of spanning trees of a connected graph $G$ is associated with the Laplacian eigenvalues, $\theta_{n} \geq \theta_{n-1} \geq$ $\cdots>\theta_{1}=0$, of the graph by the following:

$$
\prod_{j=2}^{n} \theta_{j}=n t(G) \text {. }
$$

This excludes the first Laplacian eigenvalue. Thus, as in the case of the complete graph, the eigenvalue $n-1$ of the adjacency matrix associated with $G$ will not be taken into account when considering spanning trees.

Eigenvalues have been associated with the expansion of graphs (see Hamada [7]), which motivates the idea of areas associated with a class of graphs.

If the eigen-bibalanced ratio of a class of graphs is a function of $n$, then we are able to integrate it with respect to $n$, which leads to the following definition.

Definition 14 (eigen-bibalanced ratio area of classes of graphs). We define the eigen-bibalanced ratio area of the class of graphs with respect to the eigen-pair $(a, b)$ as (see Winter and Adewusi [16])

$$
\operatorname{Ar}(\Im)^{a, b}= \begin{cases}\frac{2 m}{n}\left|\int \frac{a+b}{a b} d n\right|, & \text { if } a+b \neq 0, \\ \frac{2 m}{n}\left|\int_{a}^{b} d n\right|=\frac{2 m}{n}|2 b|, & \text { if } a+b=0,\end{cases}
$$

where $m$ is the number of edges and $n$ is the number of vertices, and $\operatorname{Ar}(\mathfrak{I})^{a, b}=0$ when $n=0,1$, or 2 .

Now we define length denoted by $B$, as

$B=2 \mathrm{~m} / n$, that is, the average degree of the vertices in $G$, and define height, denoted by $H$, as

$$
H= \begin{cases}\left|\int \frac{a+b}{a b} d n\right|, & \text { if } a+b \neq 0, \\ \left|\int_{a}^{b} d n\right|=\frac{2 m}{n}|2 b|, & \text { if } a+b=0,\end{cases}
$$

so $\operatorname{Ar}(\mathfrak{\Im})^{a, b}=B \cdot H$.

If there is more than one pair giving rise to such area, then the area of the class is $\max \operatorname{Ar}(\mathfrak{\Im})^{a_{i}, b_{i}}$ for all pairs $\left(a_{i}, b_{i}\right)$. If there is only one eigen-pair associated with the class of graphs that gives rise to the area, then the area is unique.

The height involves binding the sum of the reciprocals of the eigen-pair by its integration and the multiplication of this height by the average degree. This involves one of the most basic, yet important, combinatorial aspects of the graph and results in the term $n^{2}$ appearing in the eigen-bibalanced ratio area of some classes of graphs.

\section{Examples of Eigen-Bibalanced Classes of Graphs}

When we refer to a graph $G$ having eigen-pair balanced properties such as sum, product, bibalanced, ratio, and asymptote, we imply that $G$ belongs to a class of graphs having such 
eigen-pair properties. We will now look at various examples of eigen-bibalanced classes of graphs.

6.1. Complete Graphs. The complete graph on $n$ vertices has the unique eigen-bibalanced ratio of

$$
\begin{aligned}
r\left((n-1) K_{n}(-1)\right) & =\frac{(n-1)+(-1)}{(n-1)(-1)} \\
& =\frac{n-2}{1-n} .
\end{aligned}
$$

This depends on the order of the graph and has the unique eigen-bibalanced ratio asymptote

$$
r\left((n-1) K_{n}(-1)\right)^{\infty}=-1
$$

and eigen-bibalanced ratio area

$$
\begin{aligned}
\operatorname{Ar}\left(K_{n}\right)^{-1, n-1} & =\frac{2 m}{n}\left|\int \frac{n-2}{1-n} d n\right| \\
& =\frac{2(n(n-1) / 2)}{n}\left|\int\left[\frac{2}{n-1}-\frac{n}{n-1}\right] d n\right| \\
& =(n-1)\left|\int\left[\frac{2}{n-1}-\frac{n}{n-1}\right] d n\right| \\
& =(n-1) B,
\end{aligned}
$$

where

$$
B=\left|\int \frac{2}{n-1}-\frac{n-1}{n-1}-\frac{1}{n-1}\right| d n=n-\ln |n-1|+c .
$$

When $n=0$ we have $A=0$ so $c=0$ so that its area is

$$
\operatorname{Ar}\left(K_{n}\right)^{-1, n-1}=(n-1)(n-\ln (n-1))=(n-1) H .
$$

Note that the length of the longest path for the complete graph is $n-1$, so that $H$ in the above expression can be regarded as the height of the graph. Also, the term $\ln (n-1)$ occurs as part of the upper bound of the diameter of a graph involving the second largest eigenvalue; see Brouwer and Haemers [6].

Is this area the maximum for all classes of eigen-bibalanced graphs?

6.2. Complete Bipartite Graphs. The complete bipartite graph $K_{s, t}$ on $s+t$ vertices has the eigen-bibalanced ratio of

$$
r\left(\sqrt{s t} K_{s, t}(-\sqrt{s t})\right)=\frac{\sqrt{s t}-\sqrt{s t}}{-s t}=0
$$

which is independent of the size of the graph.

Its area is

$$
\operatorname{Ar}\left(K_{s, t}\right)^{-\sqrt{s t}, \sqrt{s t}}=\frac{2 s t}{(s+t)}|2 \sqrt{s t}|=4 \frac{(s t)^{3 / 2}}{s+t} .
$$

This attains its maximum when $s=t=n / 2$, and then the graph (the complete split bipartite graph on $n$ vertices) is $s$ regular and the area is

$$
\operatorname{Ar}\left(K_{n / 2, n / 2}\right)^{-n / 2, n / 2}=\frac{n}{2}\left|\int_{-n / 2}^{n / 2} d n\right|=\frac{n^{2}}{2} .
$$

6.3. Wheel Graphs. Wheels on $n$ vertices, containing $(n-1)$ spokes and $2(n-1)$ edges, have eigen-bibalanced ratio

$$
r\left(\frac{2+\sqrt{4+4(n-1)}}{2} W_{n} \frac{2-\sqrt{4+4(n-1)}}{2}\right)=\frac{-2}{(n-1)} .
$$

This depends on the size of the graph, so they have an eigenbibalanced ratio asymptote of 0 and eigen-bibalanced ratio area of

$$
\begin{aligned}
\operatorname{Ar} & \left(W_{n}\right)^{(2+\sqrt{4+4(n-1)}) / 2,(2-\sqrt{4+4(n-1)}) / 2} \\
& =\frac{2 m}{n}\left|\int \frac{a+b}{a b} d n\right| \\
& =\frac{2 m}{n}\left|\int \frac{2}{1-n} d n\right| \\
& =\frac{4(n-1)}{n}(\ln |n-1|+c) \\
& =\frac{4(n-1)}{n}(\ln |n-1|+c) .
\end{aligned}
$$

Now $\operatorname{Ar}\left(W_{n}\right)^{(2+\sqrt{4+4(n-1)}) / 2,(2-\sqrt{4+4(n-1)}) / 2}=0$ when $n=2$ so that $c=0$.

So

$$
\operatorname{Ar}\left(W_{n}\right)^{(2+\sqrt{4+4(n-1)}) / 2,(2-\sqrt{4+4(n-1)}) / 2}=\frac{4(n-1)}{n}(\ln |n-1|) .
$$

6.4. Star Graphs. As per Brouwer and Haemers [6], star graphs with $m$ rays of length 2 have eigenvalues $0,-1,1$, and $\pm \sqrt{m+1}$.

Using the pair $(a, b)=(-1,1)$ we obtain the ratio

$$
r\left(-1 S_{1, m P_{3}} 1\right)=\frac{0}{-1}=0
$$

and using the pair $(a, b)=(\sqrt{m+1},-\sqrt{m+1})$ we get

$$
r\left(\sqrt{m+1} S_{1, m P_{3}}-\sqrt{m+1}\right)=\frac{0}{-(m+1)}=0 .
$$

Using the class of graphs, where $(m+1)=t^{2}$ and eigen-pair $(a, b)=(\sqrt{m+1}, 1)$, we have the ratio

$$
r\left(\sqrt{m+1} S_{1, m P_{3}} 1\right)=\frac{\sqrt{m+1}+1}{\sqrt{m+1}}=\frac{t+1}{t} .
$$

Therefore the class of star graphs with $m$ rays of length 2 does not have a unique eigen-bibalanced ratio.

The area with respect to the pair $(a, b)=(-1,1)$ is

$$
\operatorname{Ar}\left(S_{1, m P_{3}}\right)^{-1,1}=\frac{2 m}{n}|2 b|=\frac{2.2 m}{2 m+1}|2|=\frac{8 m}{2 m+1}
$$

and with respect to the pair $(a, b)=(\sqrt{m+1},-\sqrt{m+1})$ is

$$
\operatorname{Ar}\left(S_{1, m P_{3}}\right)^{\sqrt{m+1},-\sqrt{m+1}}=\frac{2 m}{n}|2 b|=\frac{4 m}{2 m+1} 2 \sqrt{m+1} .
$$


Since $m=(n-1) / 2$, the areas are, respectively,

$$
\begin{aligned}
\operatorname{Ar}\left(S_{1, m P_{3}}\right)^{-1,1}=\frac{8 m}{2 m+1} & =\frac{4(n-1)}{n-1+1}=\frac{4(n-1)}{n} \\
\operatorname{Ar}\left(S_{1, m P_{3}}\right)^{-\sqrt{m+1}, \sqrt{m+1}} & =\frac{4 m}{2 m+1} 2 \sqrt{m+1} \\
& =\frac{2(n-1)}{n} 2 \sqrt{\frac{n+1}{2}} \\
& =\frac{2 \sqrt{2}(n-1)}{n} \sqrt{n+1}
\end{aligned}
$$

The greater of the two gives the area of the class of graphs; that is,

$$
\operatorname{Ar}\left(S_{1, m P_{3}}\right)^{-\sqrt{m+1}, \sqrt{m+1}}=\frac{2 \sqrt{2}(n-1)}{n} \sqrt{n+1} .
$$

6.5. Hypercube Graphs. The $p$-regular hypercube has eigen-

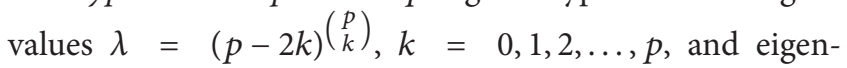
bibalanced ratio ( $k$ fixed, $n$ varying, $p \neq 2 k$, and $k \neq 0$ )

$$
\begin{aligned}
\frac{2 p-2 k}{p^{2}-2 p k} & =2 \frac{\ln n / \ln 2-k}{\ln ^{2} n / \ln ^{2} 2-2 k(\ln n / \ln 2)} \\
& =2 \ln 2 \frac{\ln n-k \ln 2}{\ln ^{2} n-2 k \ln 2 \ln n},
\end{aligned}
$$

where, since $n=2^{p}$, we have $p=\ln n / \ln 2$.

Then

$$
\operatorname{asymp}\left(\frac{2 p-2 k}{p^{2}-2 p k}\right)=0 \text {. }
$$

For $k=1$ and using the eigenvalue pair $p$ and $p-2$, the eigenbibalanced ratio area is

$$
\begin{aligned}
\operatorname{Ar}(\mathfrak{I})^{p, p-2} & =\int \frac{2 p-2}{p^{2}-2 p} d\left(2^{p}\right) \\
& =2 \ln 2 \int \frac{\ln n-\ln 2}{\ln ^{2} n-2 \ln 2 \ln n} d n .
\end{aligned}
$$

Setting $u=\ln ^{2} n-2 \ln 2 \ln n$ and rearranging, we get

$$
\ln ^{2} n-2 \ln 2 \ln n-u=0 .
$$

So

$$
\begin{aligned}
\ln n & =\frac{2 \ln 2 \pm \sqrt{(2 \ln 2)^{2}+4 u}}{2} \\
& =\ln 2+\sqrt{\ln ^{2} 2+u} \\
& \Longrightarrow n=e^{\ln 2+\sqrt{\ln ^{2} 2+u}}=2 e^{\sqrt{\ln ^{2} 2+u}} \\
& \Longrightarrow e^{\sqrt{\ln ^{2} 2+u}}=\frac{n}{2} .
\end{aligned}
$$

Also, for $u=\ln ^{2} n-2 \ln 2 \ln n$, we have

$$
\begin{aligned}
\frac{d u}{d n} & =\left[\frac{2 \ln n}{n}-\frac{2 \ln 2}{n}\right]=\frac{2}{n}(\ln n-\ln 2) \\
& \Longrightarrow d u=\frac{2}{n}(\ln n-\ln 2) d n .
\end{aligned}
$$

Now substituting the above results into (55), we get

$$
\begin{aligned}
\operatorname{Ar}(\mathfrak{I})^{p, p-2} & =2 \ln 2 \int \frac{\ln n-\ln 2}{\ln ^{2} n-2 \ln 2 \ln n} d n \\
& =2 \ln 2 \int \frac{n}{2 u} d u \\
& =\ln 2 \int \frac{n}{u} d u \\
& =\ln 2 \int \frac{e^{\ln 2+\sqrt{\ln ^{2} 2+u}}}{u} d u,
\end{aligned}
$$

$u=\ln ^{2} n-2 \ln 2 \ln n$.

Now $u>1 \Rightarrow 1 / u<1$ and $e^{\ln 2+\sqrt{\ln ^{2} 2+u}}<e^{u}$ so that

$$
\begin{aligned}
\ln 2 \int \frac{e^{\ln 2+\sqrt{\ln ^{2} 2+u}}}{u} d u & <\ln 2 \int \frac{e^{u}}{u} d u \\
& <\ln 2 \int e^{u} d u=\ln 2 e^{u} \\
& =\ln 2 e^{\ln ^{2} n-2 \ln 2 \ln n} .
\end{aligned}
$$

Although this is not a good approximation for the area of a hypercube class of graphs, it may suggest that the area of such a class of graphs is greater than that of complete graphs.

6.6. Join of Two Graphs. Taking the join of the complement of the complete graph on 2 vertices and the complete graph on $n$ vertices, we get that the resulting adjacency matrix of the graph has the conjugate pair of eigenvalues

$$
\lambda=\frac{(n-1) \pm \sqrt{(n-1)^{2}+8 n}}{2}
$$

so that their eigen-bibalanced ratio is

$$
\begin{array}{r}
r=\left(\left(\frac{(n-1)+\sqrt{(n-1)^{2}+8 n}}{2}\right)\right. \\
\left.+\left(\frac{(n-1)-\sqrt{(n-1)^{2}+8 n}}{2}\right)\right)
\end{array}
$$




$$
\begin{aligned}
& \times\left(\left(\frac{(n-1)+\sqrt{(n-1)^{2}+8 n}}{2}\right)\right. \\
& \left.\quad \times\left(\frac{(n-1)-\sqrt{(n-1)^{2}+8 n}}{2}\right)\right)^{-1} \\
& =\frac{2(n-1) / 2}{\left((n-1)^{2}-(n-1)^{2}-8 n\right) / 4} \\
& =\frac{n-1}{-2 n} \text { which tends to }-\frac{1}{2} .
\end{aligned}
$$

\subsection{Cycle Graphs}

Conjecture 15. The only class of regular graphs which are neither sum nor product eigen-pair balanced is cycles.

\section{Eigen-Bibalanced Properties of the Class of Complements of Graphs}

Theorem 16. Let $\mathfrak{I}$ be a class of eigen-bibalanced, $k$-regular graphs with eigen-pair $a, b ; a, b \neq k$ or -1 . Let $\overline{\mathfrak{J}}$ be the class of graphs consisting of the complement, $\bar{G}$, of graphs $G \in \mathfrak{I}$, where $\bar{G}$ is connected. Then, for all $\bar{G} \in \overline{\mathfrak{I}}, \bar{G}$ is $(n-1-k)$ regular, eigen-bibalanced with eigen-pair $c, d=-1-a,-1-b$, and the eigen-bibalanced ratio of $\overline{\mathfrak{I}}$ is $r(c \bar{\Im} d)=(c+d) / c d=$ $(-2-(a+b)) /(1+(a+b)+a b)$. Therefore, the class $\overline{\mathfrak{I}}$ of graphs is eigen-bibalanced.

Proof. As per Brouwer and Haemers [6], if $a, b$ are eigenvalues of $G$ and $a, b \neq k$ or -1 , then $c=(-1-a)$ and $d=(-1-b)$ are eigenvalues of $\bar{G}$.

Therefore,

Sum of the eigen-pair $(c, d)$ of $\bar{G}$

$$
\begin{aligned}
& =c+d \\
& =(-1-a)+(-1-b) \\
& =-2-(a+b),
\end{aligned}
$$

Product of the eigen-pair $(c, d)$ of $\bar{G}$

$$
\begin{aligned}
& =c d \\
& =(-1-a) \times(-1-b) \\
& =1+(a+b)+a b .
\end{aligned}
$$

Since $G \in \mathfrak{I}$ is eigen-bibalanced, then $(a+b)$ and $(a b)$ are constant integers. Therefore, $\bar{G} \in \bar{\Im}$ is eigen-bibalanced.

Therefore,

$$
r(c \widetilde{\Im} d)=\frac{c+d}{c d}=\frac{-2-(a+b)}{1+(a+b)+a b}=\text { ratio of integers. }
$$

Therefore the class $\overline{\mathfrak{\Im}}$ of graphs is eigen-bibalanced.
Corollary 17. Let $\mathfrak{\Im}$ be a class of eigen-bibalanced, $k$-regular graphs with eigen-pair $a, b ; a, b \neq k$ or -1 . Let $\bar{\Im}$ be the class of graphs consisting of the complement, $\bar{G}$, of graphs $G \in \mathfrak{J}$, where $\bar{G}$ is connected. If the asymptote of the ratio $(a+b) / a b=t$, $a, b \neq k$ or -1 and $\lim _{n \rightarrow \infty} a b=\infty$, then

$$
r(c \bar{\Im} d)^{\infty}=\operatorname{asymp}(r(c \bar{\Im} d))=\frac{-t}{t+1} .
$$

Proof. Consider the following:

$$
\begin{aligned}
r(c \bar{\Im} d) & =\frac{c+d}{c d}=\frac{-2-(a+b)}{1+(a+b)+a b} \\
& =\frac{-2 / a b-(a+b) / a b}{1 / a b+(a+b) / a b+1} .
\end{aligned}
$$

Now $\lim _{n \rightarrow \infty} a b=\infty$

$$
\begin{aligned}
\Longrightarrow r(c \bar{\Im} d)^{\infty} & =\operatorname{asymp}(r(c \bar{\Im} d)) \\
& =\lim _{n \rightarrow \infty}\left(\frac{-2 / a b-t}{1 / a b+t+1}\right)=\frac{-t}{t+1} .
\end{aligned}
$$

Corollary 18. Let $\mathfrak{I}$ be a class of eigen-bibalanced k-regular graphs with eigen-bibalanced ratio asymptote $f(t)=t$ with respect to pair $a, b$ where $a, b \neq k$ or -1 . Let $\overline{\mathfrak{I}}$ be the class of graphs consisting of the complement, $\bar{G}$, of graphs $G \in \mathfrak{I}$, where $\bar{G}$ is connected. Then the eigen-bibalanced ratio asymptote of $\overline{\mathfrak{J}}$ with respect to eigen-pair $(c, d)$ is $g(t)=-t /(t+1)$ which is an involution.

Proof. As per Corollary 17, let $g(t)=\operatorname{asymp}(r(c \bar{\Im} d))=$ $-t /(t+1)$.

Then,

$$
g\left(\frac{-t}{t+1}\right)=\frac{-(-t /(t+1))}{(-t /(t+1))+1}=\frac{t /(t+1)}{1 /(t+1)}=t
$$

so that $g[g(t)]=t$ which implies that $g(t)=g^{-1}(t)$.

Functions, which are equal to their own inverse, are called involutions, so that $g(t)$ is an involution. Classes of uniquely eigen-bibalanced graphs whose complements form a class of eigen-bibalanced graphs with an involution property are said to be involution-complementary.

Corollary 19. The involution $g(t)=-t /(t+1)$ is a solution of the differential equation

$$
\frac{d g(t)}{d t}=\frac{1}{t(t+1)} ; \quad g\left(\frac{-1}{2}\right)=1 .
$$

Proof. Since

$$
g^{\prime}(t)=\frac{-1(t+1)+t}{(t+1)^{2}}=\frac{-1}{(t+1)^{2}},
$$

then

$$
g^{\prime}(g(t))=\frac{-1}{(-t /(t+1)+1)^{2}}=-(t+1)^{2} .
$$


Also, $g(t)=-t /(t+1)$,

$$
\begin{aligned}
& \Longrightarrow t+(t+1) g(t)=0 \\
& \Longrightarrow f(t)+(t+1) g(t)=0 .
\end{aligned}
$$

Differentiating both sides, we get

$$
f^{\prime}(t)+g^{\prime}(t)(t+1)+g(t)=0 .
$$

Since $g(t)$ is an involution, $g(g(t))=t$, then differentiating both sides and recalling $f(t)=t$ we get

$$
g^{\prime}(g(t)) g^{\prime}(t)=f^{\prime}(t)=1 .
$$

Substituting (71) and (74) into (73), we get

$$
\begin{aligned}
f^{\prime}(t) & +g^{\prime}(t)(t+1)+g(t)=0 \\
& \Longrightarrow g^{\prime}(g(t)) g^{\prime}(t)+g^{\prime}(t)(t+1)+g(t)=0 \\
& \Longrightarrow-(t+1)^{2} g^{\prime}(t)+g^{\prime}(t)(t+1)+g(t)=0 \\
& \Longrightarrow g^{\prime}(t)\left[-(t+1)^{2}+(t+1)\right]+g(t)=0 \\
& \Longrightarrow g^{\prime}(t)[-t(t+1)]+g(t)=0 \\
\Longrightarrow & \frac{d g(t)}{d t}=\frac{g(t)}{t(t+1)} \\
\Longrightarrow & \frac{d y}{y}=\frac{d t}{t(t+1)},
\end{aligned}
$$

where $y=g(t)$.

Solving this variable separable equation we get

$$
\begin{aligned}
\ln |y| & =\int\left(\frac{1}{t}-\frac{1}{t+1}\right) d t=\ln |t|-\ln |t+1|+c \\
& =\ln \frac{|t|}{|t+1|}+c
\end{aligned}
$$

and $y=k(|t| /|t+1|)$ is a general solution of (69).

If $t \leq 0$ and $t+1>0$, then the solution is

$$
y=\frac{-k t}{t+1}
$$

if $y(-1 / 2)=1$, then $k=1$, so

$$
y=\frac{-t}{t+1} \text {. }
$$

In Section 6.6 we showed that $-1 / 2$ is the eigenbibalanced ratio asymptote of the class of graphs comprising the join of the complement of the complete graph on 2 vertices and the complete graph on $n$ vertices.

Note. There are other possible solutions of the general differential equation $d g(t) / d t=1 / t(t+1), g(p)=q$.

For example, when $t \geq 0 \Rightarrow t+1 \geq 0, y=t /(t+1)$ is a solution of the differential equation

$$
\frac{d g(t)}{d t}=\frac{1}{t(t+1)} ; \quad g(1)=\frac{1}{2} .
$$

\section{Properties of Eigen-Bibalanced Classes of Graphs}

Theorem 20. If a class of noncomplete graphs $\mathfrak{I}$ is eigenbibalanced with respect to the eigen-pair $(a, b),(a, b)$ are conjugate eigen-pairs arising from the quadratic $\lambda^{2}+s \lambda+t^{\prime}$, with at least one of $a, b$ positive and of the form $n+c$ (with $n$ being an integer and $c$ being negative), and the ratio $(a \Im b)=$ $(a+b) / a b$ is a function of $n$, then $t^{\prime}$ is negative and the eigenpair balanced ratio asymptote lies on the interval $[-1,0]$.

Proof. Let the conjugate eigen-pair $(a, b)$ arise from the roots of the quadratic $\lambda^{2}+s \lambda+t^{\prime}$; that is,

$$
a, b=\frac{-s \pm \sqrt{s^{2}-4 t^{\prime}}}{2} .
$$

Assume $t^{\prime}>0$, then $s \geq 2 \sqrt{t^{\prime}}$ :

$\Rightarrow t^{\prime} \geq 0$ and $s \geq 0$,

$\Rightarrow a<0$ and $b<0$, which is a contradiction of the assumption that at least one of $a, b$ is positive.

Therefore, we have shown that $t^{\prime} \leq 0$.

If $t^{\prime}=0$, then

$$
a, b=\frac{-s \pm \sqrt{s^{2}}}{2}
$$

and either $a=0$ or $b=0$, which is not allowed.

Therefore, we have shown that $t^{\prime}<0$.

So let $t^{\prime}=-t$, where $t>0$.

We have $a \leq n-1$ and $b \leq n-1$ (as these are conjugate eigenvalues of the class of noncomplete graphs $\mathfrak{\Im}$ ); $a+b=-s$; and $a b=-t$ and

$$
r(a \Im b)=\frac{a+b}{a b}=\frac{s}{t} .
$$

If $a=-b$ then the ratio $r=(a+b) / a b=0$. However, we are given that $r(a \Im b)=(a+b) / a b$ is a function of $n$, so we must have $a \neq-b$.

If $a$ and $b$ are both fixed constants, then the ratio is not a function of $n$, so we cannot have $a$ and $b$ being both fixed constants.

From (82) above, $r=s / t$. If $t=f(n), f(n)$ is of order $n$, and $s$ is a fixed constant $c$, then

$$
\operatorname{asymp}(r(a \Im b))=\operatorname{asymp}\left(\frac{s}{t}\right)=\operatorname{asymp}\left(\frac{c}{f(n)}\right)=0 .
$$

If $s=a+b$ is a function of $n$, so $t=-(a b)$ will be a function of $n$.

If both $a$ and $b$ are functions of $n$, then $a+b$ is $O\left(n^{p}\right)$ and $a b$ has $O\left(n^{q}\right)$ where $q \geq p$.

Therefore, $\operatorname{asymp}(r(a \Im b))=0$.

Now let us assume that $a=n+c>0$ and $b=k, k$ negative (as per conditions in Theorem 20).

Then if $s=n+c^{\prime}, t=k n+c^{\prime \prime},(k<0)$, and $c^{\prime}$ and $c^{\prime \prime}$ are constants, then

$$
\Longrightarrow \operatorname{asymp}(r(a \Im b))=\frac{1}{k}<0 .
$$


Since $a$ is an integer, $b=k$ must be an integer too (as they are conjugate pairs), so $k \leq-1$. Therefore,

$$
\operatorname{asymp}(r(a \Im b))=\frac{1}{k} \geq-1 .
$$

So we have proved that $-1 \leq \operatorname{asymp}(r(a \mathfrak{\Im} b))<0$.

We have therefore shown separately that asymp $(r(a \Im b))$ $=0$ and $-1 \leq \operatorname{asymp}(r(a \Im b))<0$ under different conditions.

Therefore asymp $(r(a \Im b)) \in[-1,0]$.

For the complete graph $K_{n}$, the quadratic for the complete graph, with eigenvalues $(-1, n-1)$, sum $=n-2$, and product $-(n-1)$, is

$$
\begin{aligned}
& \lambda^{2}-(n-2) \lambda-(n-1) \\
& \quad \Longrightarrow \lambda=\frac{(n-2) \pm \sqrt{(n-2)^{2}+4(n-1)}}{2} .
\end{aligned}
$$

So, $\operatorname{asymp}\left(r\left(-1 K_{n} n-1\right)\right)=(n-2) /-(n-1)=-1$.

So the eigen-bibalanced ratio asymptote of the complete graphs is -1 , which is the same as one of the eigen-pairs. If a class of graphs is eigen-bibalanced with respect to the pair $(a, b)$ and its asymptote is the same as one of the eigenpairs, then it is said to be asymptotically closed with respect to the pair $(a, b)$. Therefore, the class of complete graphs is asymptotically closed with respect to the pair $(-1, n-1)$.

Theorem 21. The eigen-bibalanced ratio areas of complete bipartite graphs, wheel graphs, and star graphs with rays of length 2 are each bounded above by the area of the complete graph.

Proof. As discussed above, the eigen-bibalanced ratio area of the complete graph is

$$
\operatorname{Ar}\left(K_{n}\right)^{-1, n-1}=(n-1)(n-\ln (n-1)) .
$$

As per Section 6.2, the eigen-bibalanced ratio area of the split complete bipartite graph is

$$
\operatorname{Ar}\left(K_{n / 2, n / 2}\right)^{n / 2, n / 2}=\frac{n^{2}}{2} .
$$

As per Section 6.3, the eigen-bibalanced ratio area of the wheel graph is

$$
\operatorname{Ar}\left(W_{n}\right)^{(2+\sqrt{4+4(n-1)}) / 2,(2-\sqrt{4+4(n-1)}) / 2}=\frac{4(n-1)}{n}(\ln |n-1|) .
$$

As per Section 6.4, the eigen-bibalanced ratio area of the star graph with rays of length 2 is

$$
\operatorname{Ar}\left(S_{1, m P_{3}}\right)^{\sqrt{m+1},-\sqrt{m+1}}=\frac{2 \sqrt{2}(n-1)}{n} \sqrt{n+1},
$$

where $m=(n-1) / 2$ and $n \geq 3$.
(1) Considering the eigen-bibalanced ratio area of the complete graph and the complete split bipartite graph, we get Table 1.

Now, for large values of $n, \operatorname{Ar}\left(K_{n}\right)^{-1, n-1}=(n-1)(n-\ln (n-$ 1)) behaves like $n^{2}$ and is an increasing function of $n$, and

$$
\operatorname{Ar}\left(K_{n / 2, n / 2}\right)^{n / 2, n / 2}=\frac{n^{2}}{2} .
$$

So we can conclude that

$$
\operatorname{Ar}\left(K_{n}\right)^{-1, n-1}>\operatorname{Ar}\left(K_{n / 2, n / 2}\right)^{n / 2, n / 2} \text { for large } n ;
$$

that is, the area of the complete graphs is greater than or equal to the area of the split complete bipartite graph.

(2) Considering the eigen-bibalanced ratio area of the complete graph and the wheel graph for $n \geq 3$, we get Table 2 .

Now, for large values of $n, A r\left(K_{n}\right)^{-1, n-1}=(n-1)(n-\ln (n-$ 1)) behaves like $n^{2}$ and is an increasing function of $n$, and

$$
\begin{gathered}
\operatorname{Ar}\left(W_{n}\right)^{(2+\sqrt{4+4(n-1)}) / 2,(2-\sqrt{4+4(n-1)}) / 2} \\
\quad=\frac{4(n-1)}{n}(\ln |n-1|)<n .
\end{gathered}
$$

So we can conclude that

$$
\begin{array}{r}
\operatorname{Ar}\left(K_{n}\right)^{-1, n-1}>\operatorname{Ar}\left(W_{n}\right)^{(2+\sqrt{4+4(n-1)}) / 2,(2-\sqrt{4+4(n-1)}) / 2} \\
\text { for large } n ;
\end{array}
$$

that is, the eigen-bibalanced ratio area of the complete graphs is greater than the area of the wheel graph for $n \geq 3$. Considering the eigen-bibalanced ratio area of the complete graph and the star graph with rays of length 2 , for $n \geq 3$, we get Table 3 .

Now, for large values of $n, \operatorname{Ar}\left(K_{n}\right)^{-1, n-1}=(n-1)(n-\ln (n-$ 1)) behaves like $n^{2}$ and is an increasing function of $n$, and $\operatorname{Ar}\left(S_{1, m P_{3}}\right)^{-\sqrt{m+1}, \sqrt{m+1}}=(2 \sqrt{2}(n-1) / n) \sqrt{n+1}$, where $m=$ $(n-1) / 2$ behaves like $2 \sqrt{2 n}$ and is an increasing function of $n$.

So we can conclude that

$$
\operatorname{Ar}\left(K_{n}\right)^{-1, n-1}>\operatorname{Ar}\left(S_{1, m P_{3}}\right)^{-\sqrt{m+1}, \sqrt{m+1}}
$$

where $m=(n-1) / 2$; that is, the eigen-bibalanced ratio area of the complete graph is greater than the area of the star graph with rays of length 2 , for $n \geq 3$.

So we can conclude that the eigen-bibalanced areas of complete bipartite graphs, wheel graphs, and star graphs with rays of length 2 are each bounded above by the area of the complete graph.

Theorem 22. If a class of graphs has eigen-bibalanced ratio

$$
r=r(a \Im b)=\frac{a+b}{a b},
$$

then ar $\neq 1$ and $b r \neq 1$.

Also, if $r$ is nonzero, the elements of the eigen-pair $(a, b)$ cannot both be $1 / r$. 
TABLE 1: Eigen-bi-balanced area of complete and complete split bipartite graphs for values on $n$.

\begin{tabular}{lcc}
\hline$N$ & Complete graph & Complete split bipartite graph \\
\hline 2 & 2 & 2 \\
3 & 4.61 & 4.5 \\
4 & 8.7 & 8 \\
5 & 14.45 & 12.5 \\
100 & 9445.08 & 5000 \\
1000 & 992100.15 & 500000 \\
10000 & 99897906.81 & 50000000 \\
\hline
\end{tabular}

TABLE 2: Eigen-bi-balanced area of complete and wheel graphs for values on $n$.

\begin{tabular}{lcc}
\hline$N$ & Complete graph & Wheel graph \\
\hline 3 & 4.61 & 1.85 \\
4 & 8.7 & 3.3 \\
5 & 14.45 & 4.4 \\
100 & 9445.08 & 18.2 \\
1000 & 992100.15 & 27.6 \\
10000 & 99897906.81 & 36.84 \\
\hline
\end{tabular}

TABLE 3: Eigen-bi-balanced area of complete and star graphs of rays of length 2 for values on $n$.

\begin{tabular}{lcc}
\hline$N$ & Complete graph & Star graph with rays of length 2 \\
\hline 3 & 4.61 & 3.77 \\
5 & 14.45 & 5.54 \\
7 & 31.25 & 6.86 \\
101 & 9639.48 & 28.28 \\
1001 & 994092.24 & 89.44 \\
10001 & 99917896.60 & 282.84 \\
\hline
\end{tabular}

Proof. Let

$$
\frac{a+b}{a b}=r(a \Im b)=r \Longrightarrow a+b=r a b .
$$

If we let $a b=y$, we get $a+b=r a b=r y$, so

$$
\begin{aligned}
& a+\frac{y}{a}=r y, \\
& \Longrightarrow a^{2}+y=r a y, \\
& \Longrightarrow y=\frac{-a^{2}}{1-a r}=a b, \\
& \Longrightarrow b=\frac{a}{a r-1}, \quad \text { for } a r \neq 1 .
\end{aligned}
$$

Swopping the roles of $a$ and $b$ we get the desired result. [11].

The following theorem can be derived from Lee and Yeh
Theorem 23. Define the class of graphs

$$
\mathfrak{I}=\overline{K_{k}} \oplus K_{n}
$$

where $k$ is fixed and $n$ varies and is greater than 1.

Then this class has eigen-pair

$$
a, b=\frac{(n-1) \pm \sqrt{(n-1)^{2}+4 n k}}{2}
$$

with eigen-bibalanced ratio

$$
r(a \Im b)=\frac{n-1}{-n k}
$$

with eigen-bibalanced ratio asymptote

$$
\operatorname{asymp}(r(a \Im b))=\frac{-1}{k}
$$

and area

$$
\operatorname{Ar}(\Im)^{a, b}=\left(\frac{n(n-1)+2 k n}{n+k}\right)\left(\frac{n}{k}-\frac{1}{k} \ln (n+1)\right) .
$$

Proof. From Lee and Yeh [11], the eigenvalue conjugate pair associated with this join is

$$
(a, b)=\frac{(n-1) \pm \sqrt{(n-1)^{2}+4 n k}}{2} .
$$

Then

$$
\begin{gathered}
\operatorname{sum}(a, b)=\frac{2(n-1)}{2}=(n-1), \\
\text { product }(a, b)=\frac{-4 n k}{4}=-k n, \\
r(a \Im b)=\frac{n-1}{-n k}, \\
\operatorname{asymp}(r(a \Im b))=\frac{-1}{k},
\end{gathered}
$$

as $n$ becomes increasingly large.

The eigen-bibalanced ratio area is (with average degree $B$ )

$$
\begin{aligned}
\operatorname{Ar}(\mathfrak{I})^{a, b} & =B\left|\int \frac{1-n}{k n} d(n+k)\right| \\
& =\frac{n(n-1)+2 k n}{(n+k)}\left|\int\left[\frac{1}{k n}-\frac{1}{k} d(n)\right]\right| \\
& =\frac{n(n-1+2 k)}{(n+k)}\left(\frac{n}{k}-\frac{1}{k} \ln n+c\right) .
\end{aligned}
$$

With $k=1$, the area must be that of the complete graph on $(n+1)$ vertices, which is, as per Section 6.1,

$$
\begin{aligned}
\operatorname{Ar}\left(K_{n+1}\right)^{-1, n} & =(n+1-1)(n+1-\ln (n+1-1)) \\
& =(n)(n+1-\ln (n)) .
\end{aligned}
$$


Hence, from (106) with $k=1$,

$$
\begin{aligned}
\operatorname{Ar}(\Im)^{a, b} & =\frac{n(n-1+2)}{n+1}\left(\frac{n}{1}-\frac{1}{1} \ln n+c\right) \\
& =\operatorname{Ar}\left(K_{n+1}\right)^{-1, n}=(n)(n+1-\ln (n)) \\
& \Longrightarrow \frac{n(n+1)}{n+1}(n-\ln n+c)=(n)(n+1-\ln (n)) \\
& \Longrightarrow \frac{n(n+1)}{n+1}(n-\ln n+c)=(n)(n-\ln (n)+1) \\
& \Longrightarrow c=1 .
\end{aligned}
$$

So,

$$
\operatorname{Ar}(\Im)^{a, b}=\left(\frac{n(n-1)+2 k n}{n+k}\right)\left(\frac{n}{k}-\frac{1}{k}(\ln n)+1\right) .
$$

Note that the complement $\bar{G}$ of any $G \in \mathfrak{I}$ above is not connected and therefore cannot be eigen-bibalanced as per the definition. Hence the class of graphs $\bar{G} \in \overline{\mathfrak{I}}$ is not eigen-bibalanced. This result applies for the class of complete graphs, complete bipartite graphs, and wheels and star graphs.

Alternatively, we could have formed the join (with $n$ vertices) $\mathfrak{I}=\overline{K_{k}} \oplus K_{n-k}$, which, by substituting $n$ with $(n-k)$ into (61), has conjugate pairs

$$
(a, b)=\frac{n-k-1 \pm \sqrt{(n-k-1)^{2}+4 k(n-k)}}{2}
$$

with ratio

$$
r(a \mathfrak{\Im} b)=\frac{k+1-n}{k(n-k)}=\frac{-(n-k)+1}{k(n-k)}
$$

which has asymptote $\operatorname{asymp}(r(a \mathfrak{\Im} b))=-1 / k$.

The area is

$$
\begin{aligned}
\operatorname{Ar}(\mathfrak{J})^{a, b}= & B\left|\int\left[-\frac{1}{k} d n+\frac{1}{k(n-k)}\right] d n\right| \\
= & B\left(\frac{n}{k}-\frac{1}{k} \ln (n-k)+c\right) \\
= & \left(\frac{(n-k)(n-k-1)+2 k(n-k)}{n}\right) \\
& \times\left(\frac{n}{k}-\frac{1}{k} \ln (n-k)+c\right) .
\end{aligned}
$$

When $k=1$, we must get the area the of the complete graph on $n$ vertices, so that

$$
\begin{aligned}
\operatorname{Ar}\left(K_{n}\right)^{-1, n-1}= & (n-1)(n-\ln (n-1)) \\
= & \left(\frac{(n-1)(n-1-1)+2(n-1)}{n}\right) \\
& \times\left(\frac{n}{1}-\frac{1}{1} \ln (n-1)+c\right)
\end{aligned}
$$

$$
\begin{aligned}
& =\left(\frac{(n-1)(n-2)+2(n-1)}{n}\right) \\
& \quad \times(n-\ln (n-1)+c) \\
& =(n-1)(n-\ln (n-1)+c)
\end{aligned}
$$

which gives $c=0$.

Hence

$$
\begin{aligned}
\operatorname{Ar}(\mathfrak{I})^{a, b}= & \left(\frac{(n-k)(n-k-1)+2 k(n-k)}{n}\right) \\
& \times\left(\frac{n}{k}-\frac{1}{k}(\ln (n-k))\right),
\end{aligned}
$$

where $\mathfrak{\Im}=\overline{K_{k}} \oplus K_{n-k}$.

Conjecture 24. The maximum eigen-bibalanced ratio area of classes of graphs on at least 6 vertices is that of the complete graph and is equal to $(n-1)(n-\ln (n-1))$.

Remark 25. The height $H=n$ of the complete split bipartite graphis greater than the height $H=n-\ln (n-1)$ of complete graphs. However multiplying by the average degree results in the complete graph having the greater area; that is,

$$
\begin{aligned}
\operatorname{Ar}\left(K_{n}\right)^{-1, n-1} & =(n-1)(n-\ln (n-1)) \\
& >\frac{n^{2}}{2}=\operatorname{Ar}\left(K_{n / 2, n / 2}\right)^{-n / 2, n / 2} .
\end{aligned}
$$

A trivial association with spanning trees and areas is given below.

From Section 6.2, the complete split-bipartite graph has area

$$
\operatorname{Ar}(G)=\frac{n^{2}}{2}=\frac{\operatorname{shad}(G)}{2 t(G)} .
$$

From Section 6.1, for the complete graph the area, in respect of the eigenvalues -1 and $n-1$, is

$$
\begin{aligned}
\operatorname{Ar}\left(K_{n}\right)^{-1, n-1} & =(n-1)(n-\ln (n-1)) \\
& =n^{2}-n \ln (n-1)-n+\ln (n-1) \\
& <n^{2}+\ln (n-1) .
\end{aligned}
$$

Now $\ln (n-1)<\ln (n)<n<2 n+1$, so

$$
\operatorname{Ar}\left(K_{n}\right)^{-1, n-1}<n^{2}+2 n+1=(n+1)^{2}=\frac{\operatorname{shad}\left(K_{n+1}\right)}{t\left(K_{n+1}\right)} .
$$

Note that, for wheels with $n$ spokes, the conjugate eigen-pair, as per Section 2.6, is

$$
(a, b)=\frac{2 \pm \sqrt{4+4 n}}{2}
$$


so that

$$
\begin{aligned}
|a+b|+|a b|= & \left|\frac{2+\sqrt{4+4 n}}{2}+\frac{2-\sqrt{4+4 n}}{2}\right| \\
& +\left|\frac{2+\sqrt{4+4 n}}{2} \cdot \frac{2-\sqrt{4+4 n}}{2}\right| \\
= & 2+n .
\end{aligned}
$$

For the join of two cycles of length $n$, there exist a pair of eigenvalues $(a, b)=2 \pm n$ so that

$$
\begin{aligned}
|a+b|+|a b| & =|2+n+2-n|+|(2+n)(2-n)| \\
& =4+\left|4-n^{2}\right| \\
& =n^{2} .
\end{aligned}
$$

See Lee and Yeh [11].

Note that, for $c(d)$, respectively, being the maximum (minimum) degrees of the vertices in the respective graphs, the wheel graph above has $|a+b|+|a b|=(2+n) \leq 3(n-1)=c d$ and the join of two cycles of length $n$ has $|a+b|+|a b|=$ $n^{2} \leq(n+2)^{2}=c d$. This suggests part (i) of the following conjecture.

For star graphs with $m$ rays of length 2 , we have eigenpair $(a, b)=(\sqrt{m+1},-\sqrt{m+1})$, and $|a+b|=\mid \sqrt{m+1}-$ $\sqrt{m+1} \mid=0$ and $|a b|=|(\sqrt{m+1})(-\sqrt{m+1})|=(m+1)$. Also $c=m$ and $d=1$, so $|a b| / d=(m+1) / 1=c+1$. So, the star graph with $m$ rays of length 2 suggests part (ii) of the conjecture below.

Conjecture 26. If a class of noncomplete graphs is eigenbibalanced with associated eigen-pair $(a, b)$ of a member of the class, the member having maximum (minimum) degree $c(d)$, respectively, then

(i) if $a+b \neq 0$, then $|a+b|+|a b| \leq c d$;

(ii) if $a+b=0$, then $|a b| / d \leq c+1$.

\section{Eigen-Bibalanced Classes of Graphs: Density}

Definition 27 (eigen-bibalanced density). The interval $[-1,0]$ is more convenient if it is a positive interval: we define the eigen-bibalanced density of a class of eigen-bibalanced graphs with asymptote asymp $(r(a \Im b))$ as

$$
\Omega_{r}(\mathfrak{\Im})=|\operatorname{asymp}(r(a \mathfrak{\Im} b))|
$$

so that the complete graph has eigen-bibalanced density 1 , which we propose as the largest density of all possible eigenbibalanced graphs (the maximum density of a class of graphs will be the largest of its densities over all its possible ratios).

Conjecture 28. The asymptotic eigen-bibalanced ratio of uniquely eigen-bibalanced classes of graphs lies in the interval $[-1,0]$ and the complete graph is the only graph which is asymptotically closed with respect to its unique eigen-pair. The density lies on the interval $[0,1]$ with the largest density being that of complete graphs, which equals 1 .

\section{Eigen-Bibalanced Classes of Graphs: Energy and Asymptotes}

There is much research on the energy of a graph - it is related to the total $\pi$-electron energy in a molecule represented by a (molecular) graph (see Adiga et al. [17]).

Definition 29 (energy of a graph). The energy of a graph with adjacency matrix $A$ with eigenvalues $\lambda_{1} \geq \lambda_{2} \geq \cdots \geq \lambda_{n}$ is

$$
E^{A}=\sum_{i=1}^{n}\left|\lambda_{i}\right|
$$

See Stevanoviç [18].

If we have a class of eigen-bibalanced graphs, is there a way of determining if the asymptotic ratio has an effect on the energy of a graph? It may be possible by assigning this asymptotic value to the vertices of the graph as, for example, a weight of a loop on a vertex; see Adiga et al. [17]. This suggests the following definition.

Definition 30 ( $r$-asymptotic eigen-bibalanced matrix). The $r$ asymptotic eigen-bibalanced matrix $C_{r}^{\infty}=\left(c_{i j}\right)$, associated with the adjacency matrix $A=\left(a_{i j}\right)$ of $G$ on $n$ vertices with an eigen-bibalanced ratio asymptote $r(a \Im b)^{\infty}$, is defined as

$$
c_{i j}= \begin{cases}a_{i j} ; & i \neq j \\ \operatorname{deg}\left(a_{j}\right)+r(a \Im b)^{\infty} ; & i=j .\end{cases}
$$

If $G$ is $k$-regular and $A$ has eigenvalues $k=\lambda_{1} \geq \lambda_{2} \geq \cdots \geq$ $\lambda_{n}$, then the eigenvalues of $C_{r}^{\infty}$ are

$$
\begin{aligned}
2 k+r(a \Im b) & =\lambda_{1} \geq \lambda_{2}+k+r(a \Im b) \\
& \geq \cdots \geq \lambda_{n}+k+r(a \Im b) .
\end{aligned}
$$

In particular, if $r=0$ the $C_{0}^{\infty}$ is the signless Laplacian matrix.

Definition 31 ( $r$-asymptotic eigen-bibalanced energy). The energy of the r-asymptotic eigen-bibalanced matrix $C_{r}^{\infty}=C$, associated with the graph $G$ on $n$ vertices and $m$ edges, with eigenvalues of $C_{r}^{\infty}=C$ being $\lambda_{1} \geq \lambda_{2} \geq \cdots \geq \lambda_{n}$, is

$$
E^{C_{r}^{\infty}}=\sum_{i=1}^{n}\left|\lambda_{i}-\frac{2 m}{n}\right| \text {. }
$$

See Stevanoviç [18].

If $r=0$, then we get the energy of the signless Laplacian matrix.

If $r \neq 0$, such as for the complete graph $K_{n}$ on $n$ vertices and $n(n-1) / 2$ edges, then its $(-1)$-asymptotic eigen-bibalanced energy is found as follows.

As per Section 2.1, the eigenvalues of $K_{n}$ are $(n-1)^{1}$, $(-1)^{n-1}$ so that the eigenvalues of $C_{-1}^{\infty}$ are

$$
\begin{aligned}
& \lambda_{1}=2(n-1)-1=2 n-3 \quad \text { once } \\
& \lambda_{2}=-1+(n-1)-1=n-3 \quad(n-1) \text { times }
\end{aligned}
$$


so that the $r$-asymptotic eigen-bibalanced energy of $G$ (with eigen-pair $(a, b))$ is

$$
\begin{aligned}
E^{C_{-1}^{\infty}}= & \sum_{i=1}^{n}\left|\lambda_{i}-\frac{2 m}{n}\right|=\left|(2 n-3)-\frac{2 n(n-1)}{2 n}\right| \\
& +(n-1)\left|(n-3)-\frac{2 n(n-1)}{2 n}\right| \\
= & |(2 n-3)-(n-1)| \\
& +(n-1)|(n-3)-(n-1)| \\
= & |(n-2)|+(n-1)|(-2)| \\
= & n-2+2 n-2 \\
= & 3 n-4 .
\end{aligned}
$$

This energy is greater than the normal energy $E^{A}=2 n-$ 2 of a complete graph on a large number of vertices. This asymptotic energy can be regarded as the eigen-pair balanced energy associated with the graph $G$ as the order of $G$ becomes increasingly large.

\section{Eigen-Bibalanced Classes of Graphs: Matrix Ratio}

Definition 32 (matrix eigen-bibalanced ratio equation). Let $A$ be the adjacency matrix of a graph $G \in \mathfrak{J}$, where $\mathfrak{I}$ is a eigen-bibalanced class of graphs. If the eigenvectors $v_{1}, v_{2}$, associated with the eigen-pair $(a, b)$, have unit length, then one has the matrix eigen-bibalanced ratio equation:

$$
\frac{\underline{v}^{t} A \underline{v_{1}}+\underline{v_{2}} A \underline{v_{2}}}{\underline{v_{1}^{t}} A \underline{v_{1} v_{2}}{ }^{t} A \underline{v_{2}}}=\frac{a+b}{a b} \text {. }
$$

For example, if $\mathfrak{I}$ is the class of complete graphs, $K_{n}$, then $A\left(K_{n}\right)$ has eigenvalues $n-1$ and -1 , with eigenvectors of unit length $\underline{v_{1}}=1 / \sqrt{n}(1,1, \ldots, 1)$ and $\underline{v_{2}}$ (which is the unit eigenvector associated with the second eigenvalue of -1 ); then

$$
\frac{{\underline{v_{1}}}^{t} A \underline{v_{1}}+\underline{v_{2}}{ }^{t} A \underline{v_{2}}}{\underline{v_{1}} A \underline{v_{1} v_{2}^{t}} A \underline{v_{2}}}=\frac{n-1+(-1)}{(n-1)(-1)}=\frac{n-2}{1-n} \text {. }
$$

This is an original definition, and is interesting along with other ratios of matrices which have been defined and investigated over time, for example, the Rayleigh ratio: $R(H, \underline{x})=$ $\underline{x}^{T} H \underline{x} / \underline{x}^{T} \underline{x}$, for any $(h x h)$ matrix $H$ and any $(h x 1)$ vector $\underline{x}$.

\section{Conclusion}

In this paper we used the ideas of integral eigenvalues and conjugate eigen-pairs to introduce the new idea of eigen-sum and eigen-product balanced properties of graphs, involving a pair of nonzero distinct eigenvalues $a$ and $b$. The fact that these attributes were nonzero, together with the idea of robustness, provided the motivation for the definition of the eigen-bibalanced ratio of classes of graphs, which allowed for the definitions of area and asymptotic ratio of classes of graphs. We found areas and asymptotes of known classes of graphs and it appears that complete graphs have the largest area and the asymptotes of all uniquely eigen-bibalanced classes of graphs may belong to the interval $[-1,0]$. Future investigation may also include classes of eigen-bibalanced graphs whose complement class is also eigen-bibalanced.

\section{Conflict of Interests}

The authors declare that there is no conflict of interests regarding the publication of this paper.

\section{References}

[1] J. M. Harris, J. L. Hirst, and M. Mossinghoff, Combinatorics and Graph Theory, Undergraduate Texts in Mathematics, Springer, New York, NY, USA, 2nd edition, 2008.

[2] J. Sarkar and A. K. Mukherjee, "Graphs with reciprocal pairs of eigenvalues," Molecular Physics, vol. 90, no. 6, pp. 903-907, 1997.

[3] J. R. Dias, "Constant-isomer benzenoid series and their topological characteristics," Theoretica Chimica Acta, vol. 77, no. 3, pp. 143-162, 1990.

[4] Y. Aimei, L. Mei, and T. Feng, "New Upper Bounds for the Energy of Graphs," 2005, http://www.orsc.edu.cn/usc/.

[5] E. R. van Dam, Regular Graphs with Four Eigenvalues, Tilburg University, 1995.

[6] A. E. Brouwer and W. H. Haemers, Spectra of Graphs, Springer, New York, NY, USA, 2011.

[7] M. Hamada, "Conjugate codes and applications to cryptology," http://arxiv.org/abs/quant-ph/0610193.

[8] A. M. Kadin, Spatial Structure of the Cooper Pair, Kluwer Academic Publishers, Princeton Junction, NJ, USA, 2005.

[9] E. J. Hinch and L. G. Leal, "Note on the rheology of a dilute suspension of dipolar spheres with weak brownian couple," Journal of Fluid Mechanics, vol. 56, no. 4, pp. 803-813, 1972.

[10] J. E. Armstrong, “The Unapologetic Mathematician," 2009, http://unapologetic.wordpress.com.

[11] S. Lee and Y. N. Yeh, "On eigenvalues and eigenvectors of graphs," Journal of Mathematical Chemistry, vol. 12, no. 1, pp. 121-135, 1993.

[12] D. A. Spielman, “Spectral Graph Theory Lecture 23," Strongly Regular Graphs, Part 1, 2009.

[13] C. Godsil and G. Royle, Algebraic Graph Theory, vol. 207 of Graduate Texts in Mathematics, Springer, New York, NY, USA, 2001.

[14] W. H. Haemers, "Matrices for graphs designs and codes," in Information Security, Coding Theory and Related Combinatorics: Information Coding and Combinatorics, D. Crnkovic and V. Tonchev, Eds., pp. 253-277, IOS Press, Amsterdam, the Netherlands, 2011.

[15] E. R. van Dam and W. H. Haemers, "Which graphs are determined by their spectrum?" Linear Algebra and Its Applications, vol. 373, pp. 241-272, 2003.

[16] P. A. Winter and F. J. Adewusi, "Tree-cover ratio of graphs with asymptotic convergence identical to the secretary problem," Advances in Mathematics: Scientific Journal, vol. 3, no. 2, pp. 4761, 2014. 
[17] C. Adiga, A. Bayad, I. Gutman, and S. Srinivas, "The minimum covering energy of a graph," Kragujevac Journal of Science, vol. 34, pp. 39-56, 2012.

[18] D. Stevanoviç, Energy of Graphs. A Few Open Problems and Some Suggestions, University of Nis, Nišs, Serbia, 2006. 


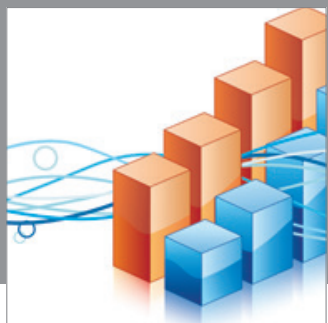

Advances in

Operations Research

mansans

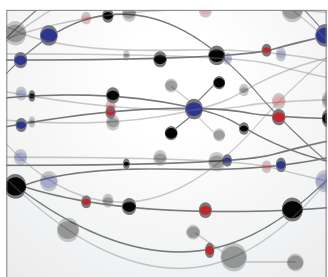

The Scientific World Journal
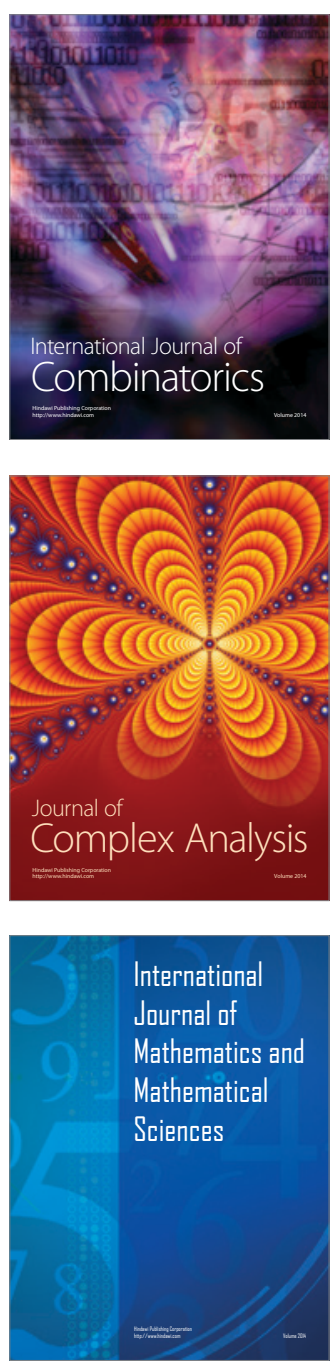
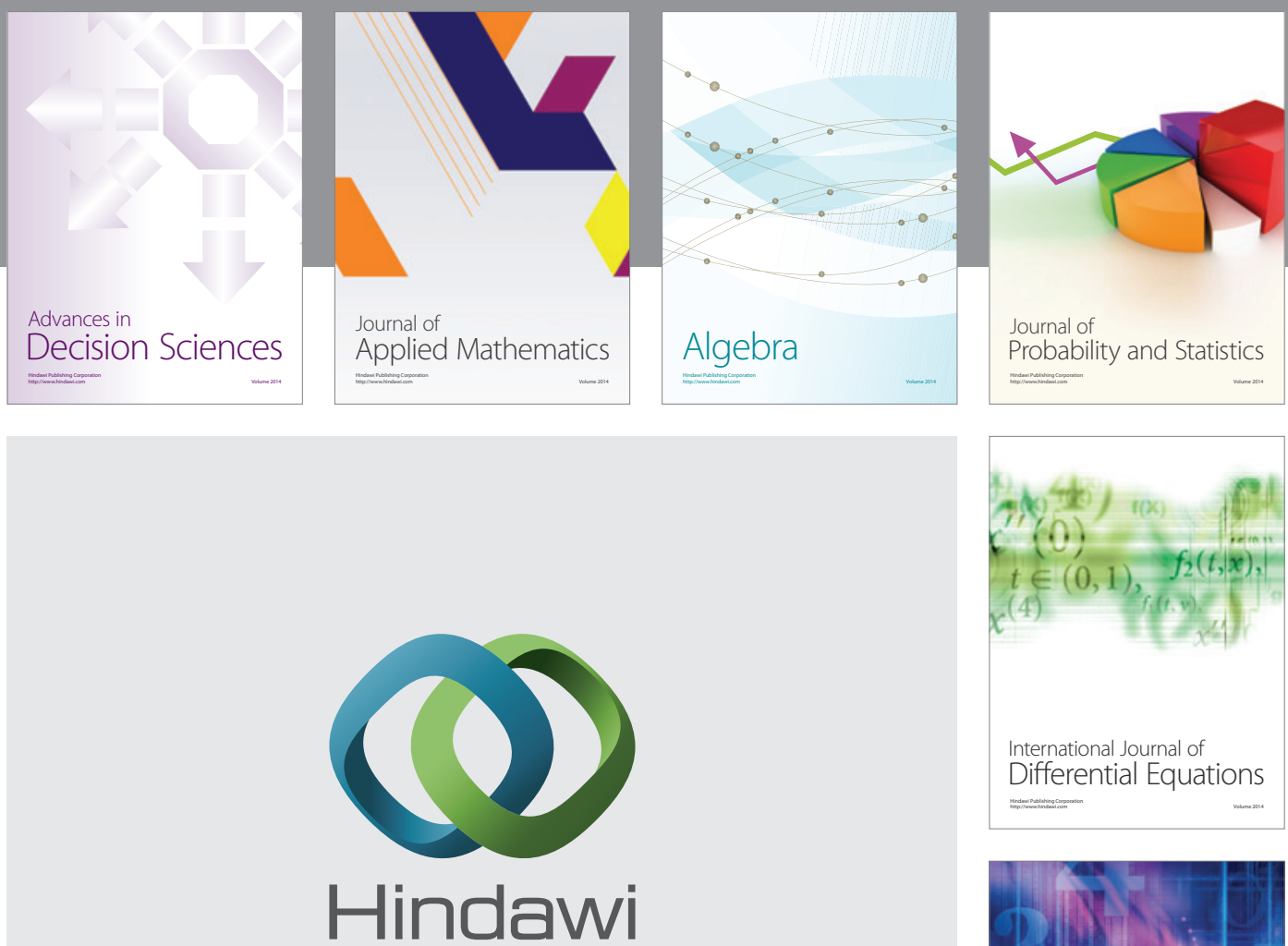

Submit your manuscripts at http://www.hindawi.com
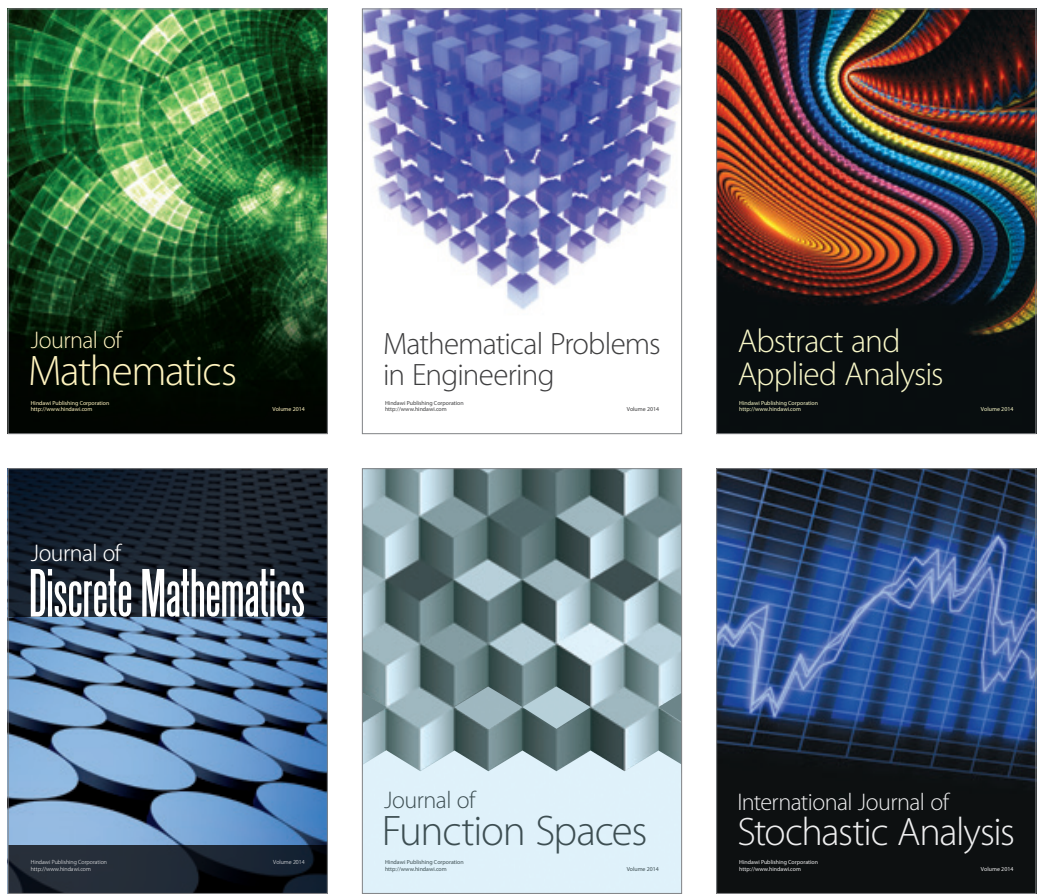

Journal of

Function Spaces

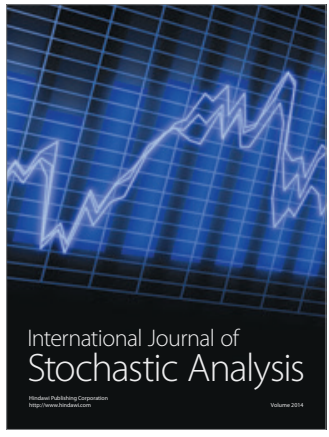

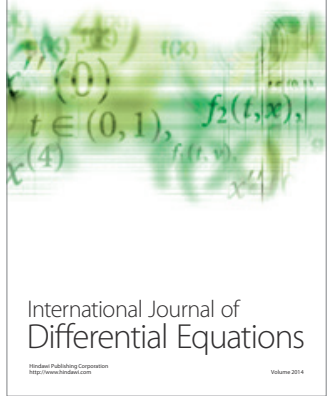
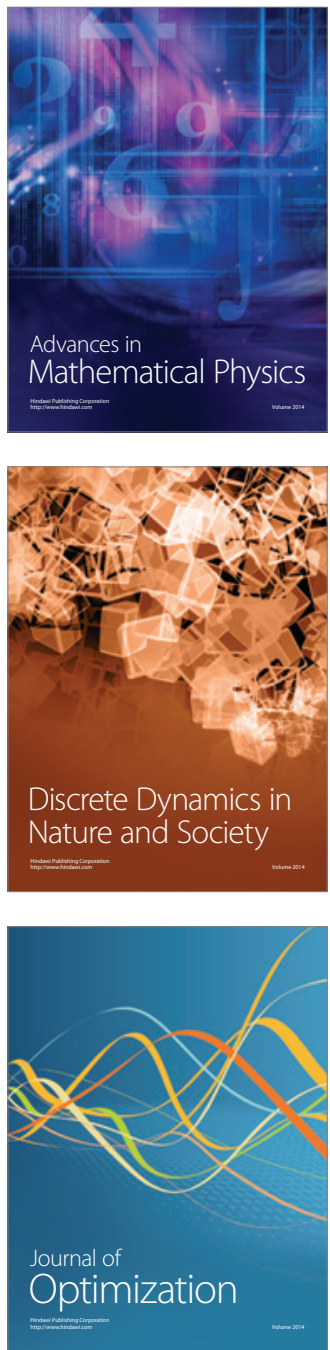